- INNOVATIVE IDEA

Volume 9 Issue 22017

DOI: 10.21315/eimj2017.9.2.7

ARTICLE INFO

Submitted: 23-01-2017

Accepted: 21-03-2017

Online: 30-06-2017

\section{Collaboration in Clinical Reasoning for e-Health Environment}

\author{
Teldja Gherib, Bouhadada T
}

Department of Computer Science, University Badji-Mokhtar, Annaba, ALGERIA

To cite this article: Gherib T, Bouhadada T. Collaboration in clinical reasoning for e-health environment. Education in Medicine Journal. 2017;9(2):55-63. https://doi.org/10.21315/ eimj2017.9.2.7

To link to this article: https://doi.org/10.21315/eimj2017.9.2.7

\title{
ABSTRACT
}

The medical education has for objective to help students to learn and reason like doctors. Many faculties of medicine have implemented teaching and learning activities like the clinical reasoning learning (CRL) sessions, for improving students' clinical reasoning skills. The ideal conditions for the organisation of these CRL sessions being difficult to meet, collaborative learning environment of the clinical reasoning at distance is an alternative effective pedagogical which can complement the shortcomings of the classical education. In this paper, we describe a collaborative learning environment, clinical reasoning, in proposing a model which allows you to make explicit an implicit process to help on the one hand, the learners to structure their thinking and approach, to better understand and interpret the elements of a clinical situation and to lead to situations rare. On the other hand, help teachers to better support the clinical reasoning of their trainees.

Keywords: Clinical reasoning, CRL, Medical diagnostic, Collaborative learning, Medical pedagogy, Collaborative environment, Distance learning

Gherib T, Department of Computer Science, Group of Research in E-learning (GreLearn), Laboratory of Research on Computing (LRI), University Badji-Mokhtar, Annaba, Algeria | Email: gheribinfo@yahoo.fr

\section{INTRODUCTION}

Although it is at the heart of the medical training, clinical reasoning is not easy to teach. It is so implicit that its appropriation during undergraduate training is sometimes difficult (1). It is also complex and its development remains unknown (2). Clinical reasoning does not end with establishing a diagnosis, it still needs to be pursued during the development of the intervention plan (3). This reasoning that will allow the doctor to make the best choice for a good diagnosis. One of the main goals in medical studies is to develop clinical reasoning skills (4). Collaborative learning methods of medical diagnosis constitutes a pedagogical alternative which allows students in medicine to improve their clinical reasoning. The clinical reasoning learning (CRL) is a pedagogical tool which teaches the student to imitate the behaviour of the expert, and promote their learning of the clinical reasoning; CRL is a collaborative and situated learning method in small groups, based on the simulation of a medical consultation. However, during the training periods, the physical distance that separates teachers and their students, makes it difficult for the organisation of these CRL sessions.

To simulate the CRL sessions at distance, we propose a model for collaborative learning supporting coordination and 
communication between a group of learners and a tutor remote geographically, which share a common task of the development of the medical diagnosis in synchronous mode. Our work is based on cognitive research accomplished in the context of the medical pedagogy, as well as on Collaborative Informational Behaviour (CIB) researches.

\section{State of the Art}

The clinical reasoning is in the heart of the professional competence of doctors (5, 6, $7,8,9)$. Higgs and Jones describe it as "A set of processes of thinking and making decisions allowing the clinician to choose the most appropriate actions in a specific context of resolution of health problems, and based on a set of cognitive skills, meta-cognitive, emotional, reflexive, and relational" (8).

There are three types of processes of reasoning used by clinicians in their diagnostic approaches (10):

1. The analytical process (hypotheticodeductive): "reflexive" or "rational" clinicians analyse rigorously and carefully the relationship between the signs, symptoms and diagnostic hypothesis.

2. Processes non-analytiques: are unconscious and automatic.

3. The mixed process: "The dual process theory", a theory that combines the two processes.

In this paper, we merely address the complex process of reasoning "the dual process theory". It is a question here of a mixed strategy where the clinician formula a hypothesis using a not-analytic strategy and then confirm it using an analytic strategy $(11,12)$. Using a mixed method during the analysis of a clinical case seems to the most likely mechanism to explain the approach of the clinician (1).

\section{The learning and teaching of clinical reasoning}

The teaching and learning of the clinical reasoning are at the heart of the medical expertise, and therefore in the center of the medical education (13). Reflective practice of Johns (14) as well as the theory of Benner (15) emphasise the importance of the development of knowledge from the educational experience (16). For Kassirer, the participation of a supervisor increases the value of an experience (17). Chamberland develops an approach to support the clinical reasoning learning: The CRL sessions within a small group of students supervised by an experienced clinician. The approach allows students to learn how to adopt effective strategy to reveal important data and to interpret them and to the extent of their discovery. It also allows to expose the intermediate stages of the reasoning process, with the help of a teacher. It is structured by critical stages such as the formulation of the problem, the relevance of the emitted hypothesis or the final synthesis that allows to highlight the key points of the strategy followed and to prepare the transfer of learnings to other clinical cases (18). A model capturing the richness and complexity of clinical reasoning processes would therefore be very useful to inform teaching, learning and assessment (19).

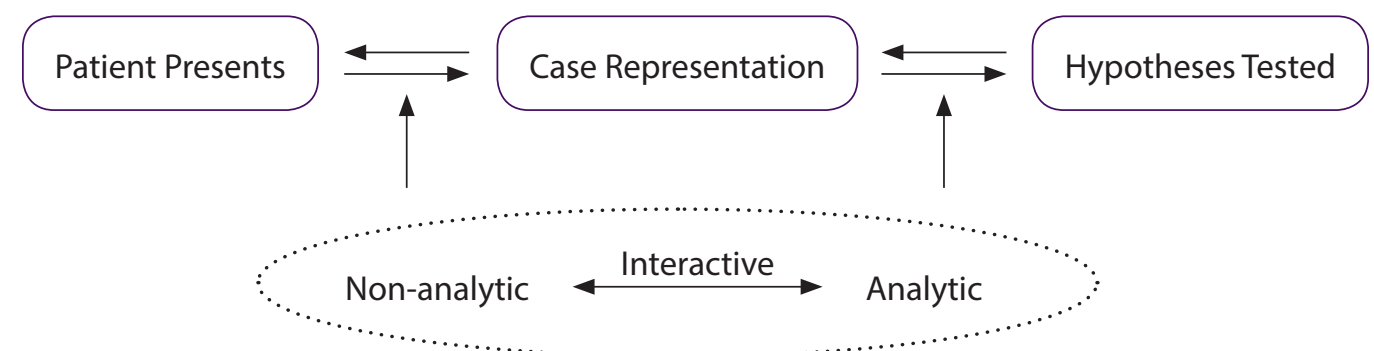

Figure 1: A combined model of clinical reasoning by Eva (12). 
Nendaz and other researchers offer different principles from cognitive psychology that allow to reflect in effective teaching activities for clinical reasoning learning $(20,21,22)$.

These principles include to:

1. Facilitate the reasoning hypotheticodeductive.

2. Promote the use of the dual process theory.

3. Promote the transfer of knowledge.

4. Promote the organisation and the activation of knowledge.

5. Promote a crop of clinical data relevant and discriminant analysis.

The development of the reasoning is intimately linked to the development of knowledge and their structuring in memory (13). The ability to acquire and organise its knowledge depends on our ability to learn and to be able to recall these knowledge in need (10). Several learning methods offer the use of a mapping (conceptual maps) to promote the development and understanding of the metacognition (23). The development of the clinical reasoning evolves especially when the clinicians share and interact on different points of view or even on a meaningful clinical experience (16). Cicourel had already demonstrated the importance of the cooperation of the actors in presence for the elaboration of the diagnosis (24).

The collaborative learning methods of medical diagnosis at distance are an effective pedagogical alternative which can complete the inadequacies of the classical education. Our goal is to propose a model of learning which deals with the clinical reasoning learning as a collaborative activity, and which supports the simulation of CRL sessions at distance.

\section{A COLLABORATIVE MODEL}

In this paper we describe a collaborative model that supports clinical reasoning learning as a collaborative activity between clinicians (students, teacher) geographically distant, whose purpose is to make explicit an implicit process, to help on the one hand, the teachers to better support the clinical reasoning of their trainees, and on the other hand, to help learners to structure their thinking and approach, to better understand and interpret the elements of a clinical situation and to practice for situations rare.

The model is based on the study carried out in the field of collaborative research "CIB: Collaborative Informational Behaviour" by Karunakaran. It also takes account of the research related to the clinical reasoning learning in the medical pedagogy. The study carried out by Karunakaran takes into consideration the collaborative research whose objective is to understand the behaviour of users seeking in a collaborative way of information, typically in digital environments (25). However, in the present work we have put the emphasis on collaborative research in the clinical reasoning (clinical case) in an e-health environment by the combination of two models published recently: that of a very recent model of collaboration proposed by Karunakaran in the field of CIB, and that of a model of the clinical reasoning proposed by Nendaz whose purpose is to study the importance of integrating the CRL in the initial training in medicine in order to give students an overview explicit on their reasoning (26).

The proposed model demonstrates that:

1. The clinical reasoning learning in collaboration includes a set of activities that take place in three phases: The formulation of the problem, the Collaborative Research and the use of the information. Some activities are specific to a particular phase, while others are common to all phases. The model allows explaining how these constitutive activities are related to each other, and how the organisational context is also a key element to understand the process of clinical reasoning in a group of learners. 
2. Triggers play an important role because they act as critical transition points from Individual Information Behaviour (IIB) to CIB (25). These factors are those who bring an individual having to appeal to other individuals (therefore, to collaborate) to achieve his goal effectively and/or quickly (27).

In our collaborative model, we find several types of reasoning used:

1. The reasoning procedural: considered as similar to the reasoning hypotheticodeductive.

2. The interactive reasoning: interaction and active collaboration between learners and tutor are used to discuss the different points of view.
3. The narrative reasoning: it is the ability to tell the story of the approach, summarise the essential steps of the problem.

4. The educational reasoning: which seeks to recognise clinical elements that are similar to the learnt theoretical concepts.

This model is distinguished by the two processes of reasoning: that of the tutor and the learner. In a first time for the tutor: the supervision of a group of learners, we have trained teachers "How to support a group of learners in their diagnostic approaches by simple interventions?" In a second step for learners: to clarify their thoughts, we formed them to "How to structure their thought and their approach? How to perform a more complete assessment of the situation of the patient prior to intervene?"

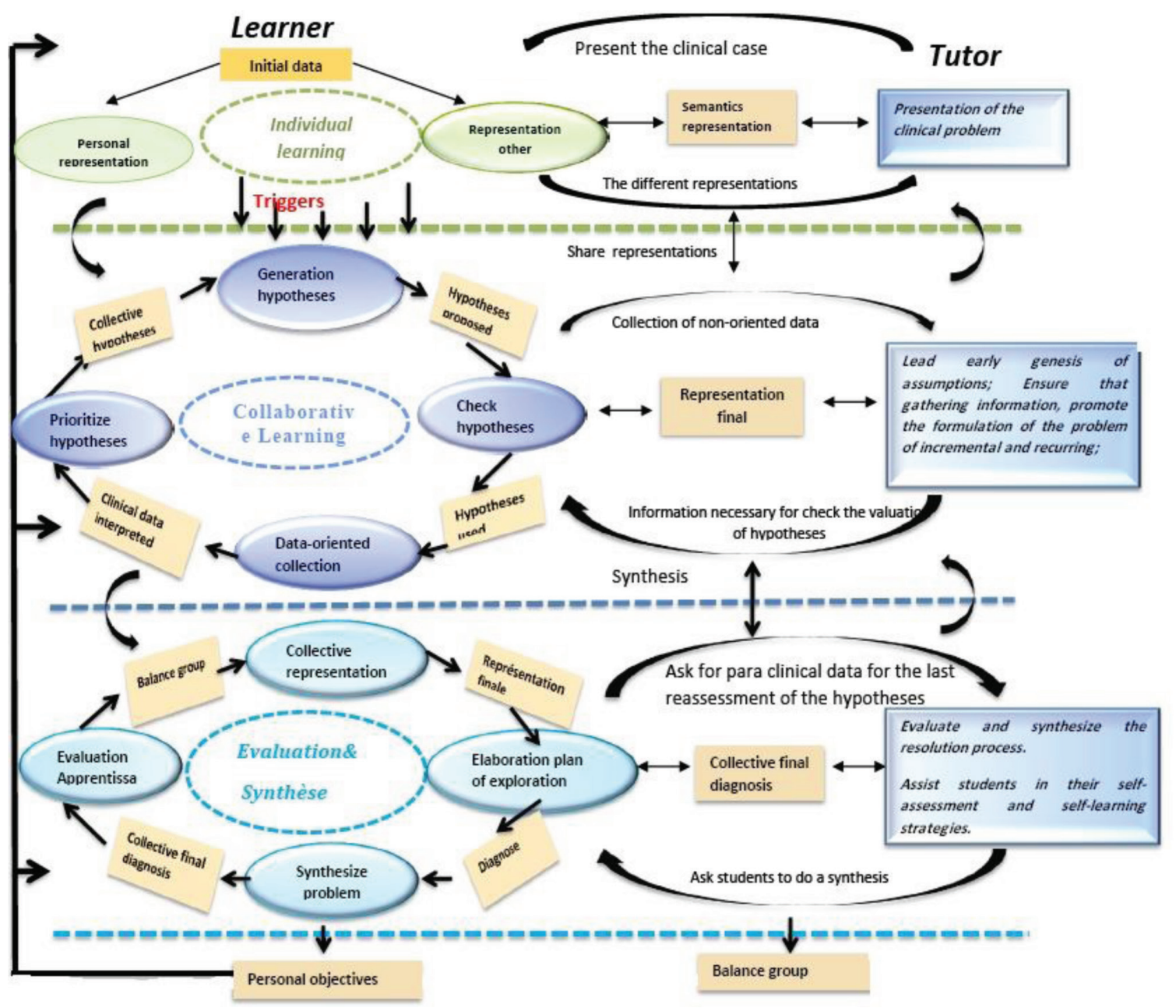

Figure 2: Collaboration in clinical reasoning for e-health environment. 
According to Audetat, the teaching clinicians can, by simple interventions, support the clinical reasoning of their trainees by concentrating not only on their knowledge and their clinical decisions, but also on their process of analysis of clinical situations (28). CRL monitor interventions will be made in a way favoured in the purpose to (29):

1. Support and encourage the early genesis of diagnostic hypotheses.

2. Ensure that information gathering, during (medical) history drawing as well as during the review and paraclinical assessment is driven by the assumptions made.

3. Encourage students to formulate the problem gradually and recurrently.

4. Ensure that students are re-evaluating in a systematic way the assumptions emitted in the term of the story, at the end of the physical examination to get a relevant diagnosis, and finally at the end of the paraclinical investigation to select the final diagnosis.

Our model allows a group of students supervised by an experimented clinician geographically distant, to treat a clinical problem. The tutor acts as a source of clinical data of the patient. After presenting the problem by the tutor, each student build his own representation. In our strategy of collaborative learning, a set of triggers, activate the transition from individual learning to collaborative learning. In this respect, the tutor invites students to verbalise explicitly, to justify their intervention to generate early diagnostic hypotheses, to ask questions that will produce new data; to reassess those hypotheses in an iterative manner and encourage the formulation of the problem in a progressive and recurring way by questions such as: what diagnostic hypotheses have you in mind? Why do you ask this questions? Is the diagnosis better specified? Are other hypotheses generated?
In the light of collective representation, learners develop an appropriate plan of exploration; each between one of them must propose a diagnosis. The tutor requires learners to evaluate and synthesise the probable diagnoses to deduce a final and collective diagnosis; at the end of the third stage in the assessment of learning, learners and the tutor assess the performance of the Group of learners, the approach of resolution, identify the errors made, analyse the participation of the Group (a balance of the Group). The tutor assists learners in their strategies of self-evaluation and selfdirected learning. Each learner strives to clarify for himself what he knows, what he will have to review or deepen. Each learner is responsible to complete alone his objectives of organisation and activation of his knowledge.

Figure 2 illustrates and explains how these sets of activities relate to the other. The model is based on three phases: A formulation of the problem, a collaborative learning process, and a synthesis and assessment phase.

\section{Phase 1: Problem Formulation Phase}

The problem formulation is the first step of our collaborative model, we can also call it "Individual learning" where the learner takes into account his cognition and metacognition to define or represent the problem. The ideal time of learning is when the student connects new information with prior knowledge network. Medical pedagogy researchers conclude that diagnostic competence pass through an early and appropriate representation in the mind of the doctor of the problem presented by the patient (23). So, it is very important to set the problem formulation as one of the key objectives of our model. This problem representation allows a semantic transformation, to give meaning to the elements, to express assumptions and to activate the relevant cognitive representations that will help lead to a diagnostic solution (1). 
In our strategy of collaborative learning, the sharing of different representations leads to a collaborative search of the clinical problem to choose the most appropriate representation. The semantic representation triggers the transition from individual learning to collaborative learning. This transition occurs by a set of "triggers." In other words, the triggers are critical points of this transition. Among these triggers, we can quote: learner's insufficient knowledge, lack of expertise, variance in representations.

\section{Phase 2: Collaborative Learning Phase}

The triggers we discussed in the previous phase lead to a "collaborative learning" phase in which several learners collaborate together to discuss the different representations shared by the previous phase to choose the most relevant.

During this whole phase, the tutor invites learners to verbalise explicitly and to justify their intervention, to generate early diagnostic hypotheses, to proceed to an oriented collection of information and to reassess these assumptions in an iterative manner.

In this learning model, the early generation of hypotheses allows learners to better structure the clinical problem in discussing the different shared representations. This iterative strategy offers learners the opportunity to collaborate and communicate their proposals whose objective is to improve their approach to solving the problem. The tutor thus encourages learners to focus exclusively on the most relevant assumptions.

This phase includes the following:

1. Generation of hypotheses: propose a hypothesis(s) to explain or resolve the situation.

2. Verification of the assumptions: justify the additional information necessary for the audit and/or evaluation of the assumptions.
3. Oriented data collection: collection and interpretation of clinical data, guided by the initial assumptions.

4. Non-oriented data collection: learners ask the tutor of the additional information necessary for the audit and/ or evaluation of the assumptions.

5. Prioritisation of assumptions: re-evaluate assumptions based on the comments and additional data and formulate others, if necessary.

During this phase the tutor supports the formulation of the problem of in a progressive and recurring way. In the end the tutor requires learners to make a synthesis of the semiological key elements in the form of a syndromic summary to formulate the problem by a collective representation in order to evaluate it.

\section{Phase 3: Synthesis \& Evaluation Phase}

In the light of the collective representation, learners establish an appropriate plan of exploration. The development of the latter and paraclinical data required are unveiled by the tutor who allows the last reassessment of the assumptions of the differential diagnosis, the selection of the final diagnosis and the development of a plan of appropriate treatment. In this step the role of the tutor is essential for learners to urge them to justify their likely diagnostic.

The tutor asks students to synthesise the problem and discuss the diagnostic path adopted by the group. This step is important to later facilitate the transfer and application of knowledge to other similar clinical situations; the tutor assists students in the organisation and the mapping of their knowledge. This phase includes the following:

1. Collective problem representation: to share the newly acquired knowledge (syndromic summary) and apply them to the resolution.

2. Development exploration plan: depending on the assumptions used, the 
group claims the tutor to give the results of further tests considered essential to progress in diagnostic reasoning.

3. Summarise the problem: the tutor asks students to synthesise the problem and discuss the diagnostic path adopted by the group to develop a collective final diagnosis.

4. Learning assessment: learners and tutor assess the performance of the group, of the solution proposed, identify the errors made, analyse the participation of learners.

Tutor assists learners in the progressive development of their strategies of selfevaluation and self-directed learning. After individual reflection, each learner strives to clarify for himself what he knows, what he will have to review or deepen. Each student is responsible for completing alone his objectives the organisation and the activation of his knowledge. In the term of this phase, the tutor recontextualise the knowledge discussed by illustrating them through additional examples.

The formalisation of these iterative processes (table, concept map, etc.) allows having a structure simple and readable that sums up most of our collaborative approach. In addition, it is an ideal for learners to update the metacognitions and cognitions.

\section{CONCLUSION AND PROSPECTS}

In this paper, we presented a collaborative model that supports the learning of clinical reasoning (CR) as a collaborative activity between clinicians (students, teachers) geographically distant and that promotes skill improvement in the clinical environment. The model is primarily dedicated to the design of collaborative learning environments for medical diagnostics, in synchronous mode. This proposal is based on the study carried out by some works in the CIB domain "Collaborative Informational Behaviour", which considers triggers as specific triggers that move an individual from a situation of individual information search to a situation of collaboration. To have a relevant learning model, we have tried to exploit the cognitive studies carried out in the field of medical pedagogy that have attempted to identify the basic principles for CRL and the factors that may influence them. However, at the present stage of research, there are still many areas of shadow that need to be clarified.

The model allows answering relevant questions in the field of teaching-learning of clinical reasoning such as:

1. How to encourage the student to make the complete assessment of the patient's situation before intervening?

2. How to facilitate the learning of abstract concepts such as clinical reasoning, resolution, clinical decision-making, critical thinking and reflexive thinking?

3. How do clinician teachers support the clinical reasoning of their trainees?

4. How to develop a "teamwork" behavioural skill?

However, the model deserves to be further refined and experimented in a real environment. This approach will certainly allow us to objectively measure the scope of the choices that have been adopted in this paper.

\section{REFERENCES}

1. Demeester A, Eymard C, Vanpee D. Learning clinical reasoning: identified difficulties for future midwives. Revue française de pédagogie. 2012; 181:43-54. https://doi.org/10.4000/rfp.3906.

2. Bourget A. Explanation of clinical reasoning: innovative methodology leading to the identification of two stages of development during the 12 months of the end of preclinical training and the beginning of clinical training for medical students. Recherches Qualitatives. 2013;32(2):320-45. 
3. Pelaccia T, Tardif J, Triby E. Comment les médecins urgentologues raisonnent-ils au regard des spécificités de leur cadre et leur mode d'exercice? [dissertation]. Français: Université de Strasbourg; 2014.

4. Ortega EM. 2004. Design of a collaborative multimedia environment for remotely managed clinical reasoning sessions [dissertation]. France: University in Rennes; 2004.

5. Loftus S. Rethinking clinical reasoning: time for a dialogical turn. Medical Education. 2012;46(12):1174-8. https://doi. org/10.1111/j.1365-2923.2012.04353.x.

6. Chamberland M, St-Onge C, Setrakian J, Lanthier L, Bergeron L, Bourget A, Mamede $\mathrm{S}$, Schmidt H, Rikers R. The influence of medical students' self-explanations on diagnostic performance. Med Educ. 2011;45(7):688-95. https://doi.org/10.1111/ j.1365-2923.2011.03933.x.

7. Schwartz A, Elstein AS. 2008. Clinical reasoning in medicine. In: Higgs J, Jones MA, Loftus S, Christensen $\mathrm{N}$, editors. Clinical reasoning in the health professions. Oxford: Butterworth-Heinemann; 1995. p. 223-34.

8. Higgs J, Jones MA. Clinical decision making and multiple problem spaces. In: Higgs J, Jones MA, Loftus S, Christensen N. Clinical reasoning in the health professions. Oxford: Butterworth-Heinemann; 2008. p. 3-18.

9. Bernard C, Jacques T, Boshuizen HP. Scripts and medical diagnostic knowledge: theory and applications for clinical reasoning instruction and research. Academic Medicine. 2000;75(2): 182-90. https://doi. org/10.1097/00001888-200002000-00020.

10. Guyete D, Rinaudo J-L. La formalisation et l'apprentissage du raisonnement clinique en formation initiale de masseur kinésithérapeute, une identification des savoirs de la pratique professionnelle. France: Departement des Sciences de l'Education, Université de Rouen; 2013.
11. Audetat M-C, Charlin B, Blais J-G. The identification and remediation of clinical reasoning difficulties in medicine (State of practices, research of tools and processes to support clinician teachers) [dissertation]. Canada: Université de Montréal; 2011.

12. Eva KW. Ce que tout enseignant devrait savoir concernant le raisonnement clinique. Pédagogie Médicale. 2005;6(4):225-34. https://doi.org/10.1051/pmed:2005027.

13. Collard A, Bourguignon J-P, Bredart S. The development of biomedical and clinical reasoning during the medical curriculum [dissertation]. Belgique: Université de Liège; 2014.

14. Johns C. Engaging reflection in practice: a narrative approach. Oxford, England: Blackwell Publishing; 2006. p. 298.

15. Benner P. 1982. From novice to expert. The American Journal of Nursing. 82(3):402$7 . \quad$ https://doi.org/10.1097/00000446198282030-00004.

16. Plante $\mathrm{H}$, Larue $\mathrm{C}$. The development of clinical reasoning for newly qualified nurses in critical care [dissertation]. Canada: Faculté des sciences infirmières, Université de Montréal; 2014.

17. Kassirer JP. Teaching clinical reasoning: casebased and coached. Acad Med. 2010;85(7): 1118-24. https://doi.org/10.1097/ ACM.0b013e3181d5dd0d.

18. Chamberland M. Les séances d'apprentissage du raisonnement clinique (ARC): description de la méthode pédagogique. Quebec, Canada: Université de Sherbrooke; 2007.

19. Charlin B, Lubarsky S, Millette B, Crevier F, Audetat M-C, Charbonneau A, Cairefon N, Hoff L, Bourdy C. Clinical reasoning processes: unravelling complexity through graphical representation. Medical Education. 2012:46(5):454-63. https://doi.org/10.1111/ j.1365-2923.2012.04242.x. 
20. Nendaz M, Charlin B, Leblanc V, Bordage G. Clinical reasoning: data from research and implications for teaching. Medical Education. 2005;6(4):235-54. https://doi. org/10.1051/pmed:2005028.

21. Regehr G, Norman GR. Issues in cognitive psychology: implications for professional education. Academic Medicine. 1996;71:988-1001. https://doi. org/10.1097/00001888-199609000-00015.

22. Tardif J. For a strategic education: the input of cognitive psychology. Montréal: Les Editions Logiques; 1992.

23. Groves $M$. Understanding clinical reasoning: the next step in working out how it really works. Medical Education. 2012:46(5):4446. https://doi.org/10.1111/j.1365-2923.2012. 04244.x.

24. Cicourel AV. Medical Reasoning. A sociocognitive approach. Paris: Seuil; 2002.

25. Karunakaran A, Reddy MC, Spence PR. Toward a model of collaborative information behavior in organizations. Journal of the American Society for Information Science and Technology. 2013 Dec;64(12):2437-51. https://doi.org/10.1002/asi.22943.
26. Nendaz $M$, Gut AM, Louis-Simonet $M$, Perrier A, Vu NV. Bringing explicit insight into cognitive psychology features during clinical reasoning seminars: a prospective, controlled study. Education for Health. 2011;24(1):496.

27. Vivian R, Dinet J. Collaborative information research : towards a user-centric system. Digital Document. 2007;10(3):136.

28. Audetat M-C, Laurin S, Sanche G. Supporting clinical reasoning trainees, to explain and clarify. The Quebec Doctor. 2014;49(1):67-9.

29. Chamberland M. Les séances d'apprentissage du raisonnement clinique (ARC): description de la méthode pédagogique. l'Université de Sherbrooke: Faculté de médecine et des sciences de la santé; 2007. 